\title{
A new species of Mycena sect. Polyadelphia from southern India
}

\section{Aravindakshan DM and Manimohan P*}

Department of Botany, University of Calicut, Kerala, 673 635, India

Aravindakshan DM, Manimohan P 2012 - A new species of Mycena sect. Polyadelphia from southern India. Mycosphere 3(2), 241-243, Doi 10.5943 /mycosphere/3/2/7

Mycena saparna sp. nov. from Kerala State, India is described, illustrated and discussed. It is placed in Mycena sect. Polyadelphia where it seems closely allied to M. foliicola reported from Madagascar.

Key words - Agaricales - Basidiomycota -Mycenaceae- mycota - taxonomy

\section{Article Information}

Received 12 April 2012

Accepted 17 April 2012

Published online 30 April 2012

*Corresponding author: P Manimohan - e-mail - pmanimohan@gmail.com

\section{Introduction}

Mycena sect. Polyadelphia Singer ex Maas Geest. comprises about 30 species described globally. Species belonging to this section are characterized by very small basidiomata growing on non-woody substrata, limited number of distant, broadly adnate lamellae, filiform stipe, absence of pleurocystidia, cheilocystidia typically covered with warts, and hyphae of both the pileipellis and stipitipellis densely ornamented with short warts. They grow mainly on fallen, decaying leaves of deciduous trees and occasionally also on herbaceous stems, fronds and rhizomes of ferns and conifer needles (Maas Geesteranus 1992, Villarreal et al. 2002). Mycena polyadelpha (Lasch) Kühner is the type species of the section. During our studies on Mycena species of Kerala State, India, we came across, on several occasions, an as yet undescribed species that belongs in this section. The fungus is described, illustrated and discussed here.

\section{Methods}

Conventional morphology based taxonomic methods were employed for this study. Microscopic observations were made on material stained with $1 \%$ aqueous solution of Congo red and mounted in $3 \%$ aqueous $\mathrm{KOH}$.
Melzer's reagent was used to observe whether the spores and tissues were amyloid or vinoid. For evaluation of the range of spore size, twenty basidiospores from each specimen cited were measured. Colour codes used in the description are from Kornerup \& Wanscher (1978). The examined collections are deposited at Kew (Mycology) Herbarium and the Kew accession numbers (e.g., K (M) 175513) are indicated. The infrageneric taxonomy of Mycena followed is that of Maas Geesteranus (1992).

\section{Results}

Mycena saparna Aravind. \& Manim. sp. nov.

MB 800054 (http://www.mycobank.org).

Fig. 1

Etymology - saparna (Sanskrit), associated with leaves.

Differing from Mycena foliicola Métrod in having subdecurrent to decurrent lamellae, tetrasporic basidia, clavate, cylindrical or sphaero-pedunculate cheilocystidia, and geographic distribution.

Basidiomata very small, delicate. Pileus 0.1-1.25 (2.5) $\mathrm{mm}$ diam., 0.5-2.75 $\mathrm{mm}$ high, broadly hemispherical with an acute or acuminate umbo when young, becoming conic 
or parabolic to cylindrico-campanulate with persisting umbo; surface off-white all over when very young, then yellowish brown (5D4) or greyish yellow (4C3) on umbo, whitish towards margin, and brownish orange (5C3) or yellowish grey (4B2) elsewhere, and finally becoming dark brown (6F7) or greyish brown (5E3) at the centre, whitish at margin, and brown (6E5) or orange grey (6B2) or brownish orange (5C3) elsewhere, sulcate-striate, dry, with fine ridges over mature pileus under a lens; margin slightly incurved and entire when young, becoming straight or nearly applanate and crenate or undulate with age. Lamellae 49, descending, subdecurrent to somewhat arcuate, off-white, up to $0.1 \mathrm{~mm}$ wide, distant, without lamellulae; edge finely torn under a lens, concolourous with the sides. Stipe 4-22 $\times$ $0.5-0.75 \mathrm{~mm}$, central, terete, tapering towards apex in all stages of development, hollow; surface translucent, whitish or orange-white (5A2), becoming brownish at extreme base, glabrous; base slightly broad, with a thin, inconspicuous basal mycelial felt. Context not conspicuous. Odour and taste not distinctive.

Basidiospores 6-9.5 $\times \quad 3-4.5$ $(6.85 \pm 0.266 \times 3.075 \pm 0.034) \mu \mathrm{m}, \mathrm{Q}=2-2.66$, $\mathrm{Qm}=2.33$, oblong-ellipsoid, thin-walled, hyaline, smooth, with a few guttules, amyloid. Basidia 7.5-17 × 5-7 $\mu \mathrm{m}$, clavate, bearing 4 sterigmata up to $5 \mu \mathrm{m}$ long, hyaline, with a few guttules. Lamella-edge sterile. Cheilocystidia crowded, 6-20.5 × 2.75-6 $\mu \mathrm{m}$, clavate, cylindrical or sphaero-pedunculate, thinwalled, hyaline, with short, conical or cylindrical excrescences $(0.75-2 \times 0.75-1 \mu \mathrm{m})$. Pleurocystidia none. Lamellar trama subregular; hyphae 2-11 $\mu \mathrm{m}$ wide, thin-walled, hyaline, vinoid in Melzer's reagent. Subhymenium pseudoparenchymatous. Pileus trama subregular; hyphae 3-32 $\mu \mathrm{m}$ wide, thinwalled, hyaline or with pale greyish brown contents, vinoid in Melzer's reagent. Pileipellis a cutis; hyphae 1-4.5 $\mu \mathrm{m}$ wide, slightly branched, slightly gelatinized, thin-walled, hyaline, with short, conical or cylindrical excrescences $\quad(0.5-2 \times 0.5-0.75 \mu \mathrm{m})$. Stipitipellis a cutis; hyphae 1.5-6 $\mu \mathrm{m}$ wide, thin-walled, hyaline, with short, conical or cylindrical excrescences $(0.5-2 \times 0.5-1 \mu \mathrm{m})$, terminal cells not inflated and not modified in any way. Caulocystidia none. Stipe trama strongly vinoid in Melzer's reagent. Clamp connections seen on all hyphae.

Habitat - On decaying leaves of various dicotyledonous plants, scattered or in groups, July, September-October.

Known distribution - Calicut and Malappuram Districts of Kerala State, India.

Material examined - INDIA, Kerala State, Malappuram District, Calicut University Campus, 5 Jul 2007, D.M. Aravindakshan DM153 (K(M) 175513, holotype); ibid., 18 Jul 2007, D.M. Aravindakshan DM170 (K(M) 175514); 20 Oct 2010, D.M. Aravindakshan DM509; Kozhikode District, Koyilandy, Poyilkavu, 26 Sep 2009, D.M. Aravindakshan DM341 (K(M) 175515).

\section{Discussion}

Mycena saparna is characterized by minute, fragile, foliicolous basidiomata; conico-campanulate pileus with a prominent acuminate umbo; subdecurrent to decurrent lamellae without lamellulae in between; filiform, glabrous stipe with an inconspicuous basal mycelial felt; oblong-ellipsoid, amyloid basidiospores; cheilocystidia with short conical excrescences; and hyphae of both pileipellis and stipitipellis showing short conical excrescences. In the current infrageneric classification (Maas Geesteranus 1992), this species is placed in sect. Polyadelphia owing to the following characters: ellipsoid and amyloid basidiospores, lamella-edge concolourous with the sides, stipe arising from a basal patch of mycelium, hyphae of the pileipellis more or less densely covered with short simple excrescences, clavate cheilocystidia that are covered with excrescences and not embedded in a gelatinous matter, and absence of pleurocystidia. However, no other species so far described from sect. Polyadelphia agrees fully morphologically with that of M. saparna. Keys to the Northern Hemisphere species of the section by Maas Geesteranus (1992) and Villarreal et al. (2002) do not lead the present species to any known species. Similarly, it is not fully similar to any of the species of sect. Polyadelphia described from Brazil (Maas Geesteranus \& de Meijer 1997), Australia (Grgurinovic 2003), New Zealand (Segedin 1991) or Malaysia (Corner 1994). 


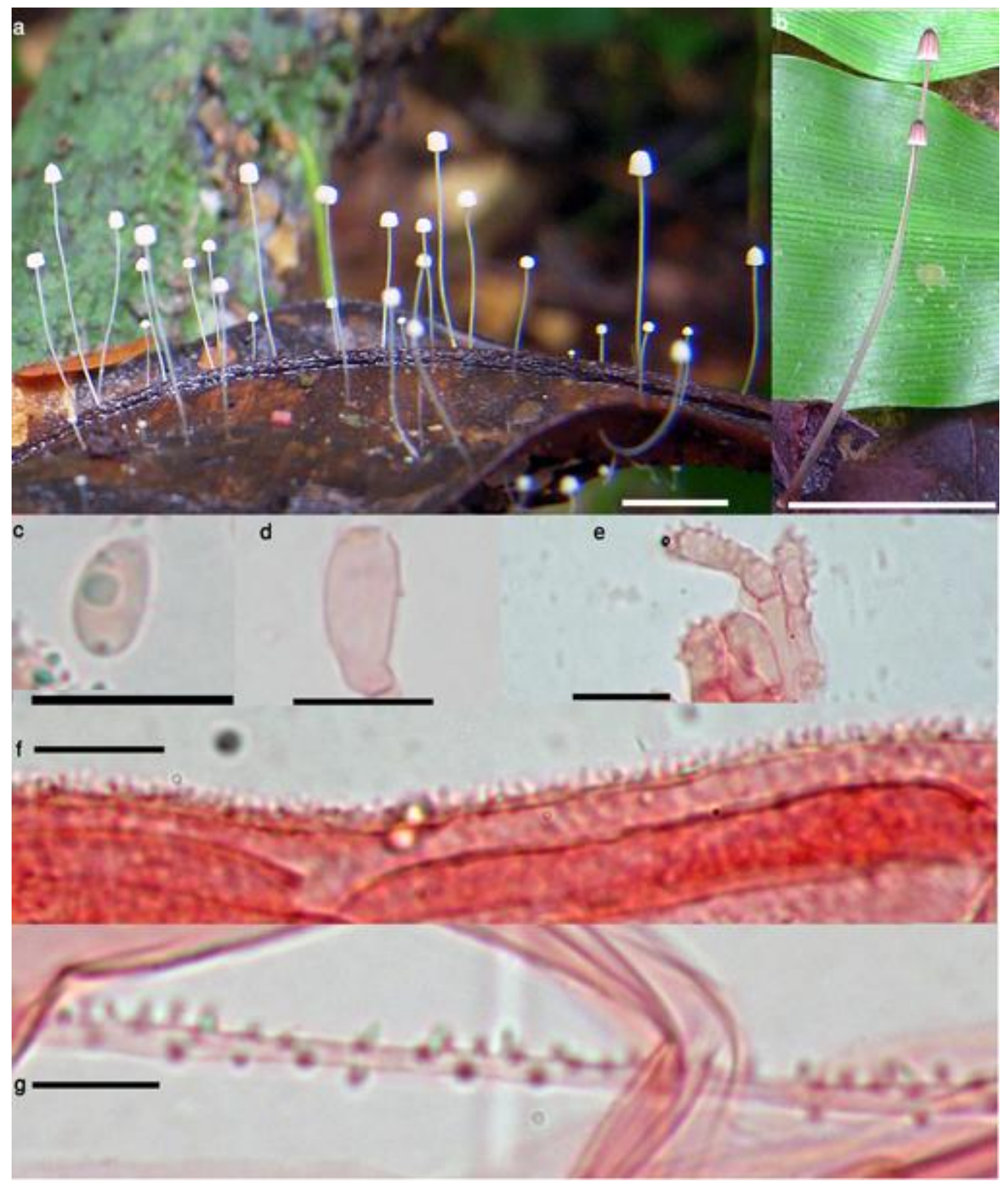

Fig. 1 - Mycena saparna. a, b Basidiomata. c Basidiospore. d Basidium. e Cheilocystidia. f hyphae of the pileipellis. $\mathbf{g}$ hyphae of the stipitipellis. Bars: $\mathrm{a}, \mathrm{b}=1 \mathrm{~cm} \mathrm{c}-\mathrm{g}=10 \mu \mathrm{m}$.

Extensive searches in the literature on mycenas, however, revealed that $M$. saparna is strikingly similar to $M$. foliicola Métrod in several features. Mycena foliicola was originally described from Madagascar by Métrod (1949). It is a very small, delicate, and foliicolous species that has not been reported since its original discovery. Métrod's description and illustrations of the species are scanty but adequate for comparison. Although Métrod (1949) placed $M$. foliicola in sect. Granulatae Lange subsect. Insititiae Fries, a 
reassessment of its features indicate that it is a good representative of sect. Polyadelphia in the modern infrageneric classification of the genus. In fact, the only species with which Métrod (1949) compared $M$. foliicola was $M$. polyadelpha. Both $M$. foliicola and $M$. saparna have very small, fragile, foliicolous basidiomata with conico-campanulate pileus, an acuminate umbo, and similar-sized basidiospores. However, there are both microscopic and macroscopic differences. The lamellae are ascending and broadly adnate, basidia are bisporic and the cheilocystidia are ovoid in $M$. foliicola. These differences along with the geographical difference are considered sufficient for the erection of a new species.

\section{Acknowledgements}

We thank Mr K.N. Anil Raj for his help in collecting Mycena specimens.

\section{References}

Corner EJH. 1994. Agarics in Malesia I Tricholomatoid. II Mycenoid. Beihefte zur Nova Hedwigia_109, 1-271.
Grgurinovic CA. 2003. The genus Mycena in South-Eastern Australia. Fungal Diversity Press, Hong Kong.

Kornerup K, Wanscher A. 1978. Methuen handbook of colour, 3rd edn. Methuen, London.

Maas Geesteranus, RA. 1992. Mycenas of the Northern Hemisphere. II. Conspectus of the Mycenas of the Northern Hemisphere. North-Holland, Amsterdam.

Maas Geesteranus, RA, de Meijer AAR. 1997. Mycenae Paranaenses. North-Holland, Amsterdam.

Métrod G. 1949. Les Mycènes de Madagascar (Mycena, Corrugaria, Pterospora). Laboratoire de Cryptogamie du Museum National d'Histoire Naturelle, Paris.

Segedin BP. 1991. Studies in the Agaricales of New Zealand: some Mycena species in sections Longisetae, Polyadelphia, Rubromarginatae, Galactopoda, Lactipedes, and Calodontes. New Zealand Journal of Botany 29, 43-62.

Villarreal M, Arenal F, Rubio, V. 2002. Mycena pinophila, a new species of Mycena section Polyadelphia from Spain. Mycotaxon 84, 331-336. 